\title{
L'agrément des faits : le retour de la « vertu » de suauitas dans la rhétorique humaniste de la narration (1450-1710)
}

Jean Lecointe

\section{(2) OpenEdition}

Journals

Édition électronique

URL : https://journals.openedition.org/rhetorique/1314

DOI : $10.4000 /$ rhetorique. 1314

ISSN : 2270-6909

Éditeur

UGA Éditions/Université Grenoble Alpes

Édition imprimée

ISBN : 978-2-37747-345-8

\section{Référence électronique}

Jean Lecointe, «L'agrément des faits : le retour de la « vertu » de suauitas dans la rhétorique humaniste de la narration (1450-1710) », Exercices de rhétorique [En ligne], 18 | 2022, mis en ligne le 22 février 2022, consulté le 26 février 2022. URL : http://journals.openedition.org/rhetorique/1314 ; DOI : https://doi.org/10.4000/rhetorique.1314

Ce document a été généré automatiquement le 26 février 2022.

\section{c) (i) (2) (2)}

Les contenus de la revue Exercices de rhétorique sont mis à disposition selon les termes de la Licence Creative Commons Attribution - Pas d'Utilisation Commerciale - Partage dans les Mêmes Conditions 4.0 International. 


\section{L'agrément des faits : le retour de la « vertu » de suauitas dans la rhétorique humaniste de la narration (1450-1710)}

Jean Lecointe

1 La doctrine des « vertus » - virtutes ou $\dot{\alpha}_{\rho \varepsilon \tau} \tilde{\alpha}_{l}$ (aretai) - de la narration constitue un des piliers de la théorie rhétorique antique relative à la conduite du récit dans le discours oratoire. Constituée vraisemblablement, sous sa forme la plus simple, de la tripartition brièveté-clarté-vraisemblance (ou plutôt «fiabilité »), et ce, dès les origines de la rhétorique, cette doctrine a ensuite connu une extraordinaire fortune dans les traités antiques, tant grecs que latins, et peut-être plus encore dans les rhétoriques humanistes. Le «traditionnisme" de la pensée humaniste en est une des causes principales, et, a priori, on pourrait discuter l'intérêt d'énumérer une interminable série de reprises, déclinées plus ou moins à l'identique. Ce serait là une impression trompeuse. Même dans le cadre de la réactualisation de la doctrine la plus canonique, la rhétorique humaniste est capable de faire preuve à l'occasion d'une certaine créativité. Mais, surtout, vers le milieu du $\mathrm{XvI}^{\mathrm{e}}$ siècle, il est possible d'assister à une spectaculaire mutation théorique, non pas certes à partir d'une innovation pure et simple, mais à la faveur de la réintroduction dans l'exposé d'une formule quadripartite, cette fois, intégrant une quatrième "vertu ", la suauitas.

2 Cette quadripartition, le Cicéron de la maturité en avait déjà proposé la formule, dans ses Divisions de l'art oratoire, mais, de facto, elle était demeurée longtemps plutôt marginale dans le discours rhétorique dominant. C'est donc à l'analyse de cette redécouverte de la suauitas, des précédents antiques de la doctrine, et de la possible signification de sa résurgence au sein de la rhétorique humaniste que nous voudrions nous attacher ici ${ }^{1}$. 


\section{Les « vertus de la narration » dans la rhétorique antique}

3 La rhétorique antique accorde la plus grande importance à la définition des "vertus " du discours, qu'il s'agisse de qualités générales du style ou de qualités plus spécifiques à certains types de discours, notamment à la narration. Il semble bien, d'ailleurs, que ce soit à ce propos même de la narration, envisagée principalement comme seconde partie du plaidoyer judiciaire, consécutive à l'exorde, que la doctrine des qualités du style $\dot{\alpha}_{\rho \varepsilon \tau} \alpha_{\alpha} \lambda \lambda_{\varepsilon} \xi \varepsilon \omega \varsigma$ (aretai lexeôs) - ait amorcé son développement ${ }^{2}$. Attribuée par certains au rhéteur sicilien Corax, la doctrine était probablement déjà établie chez Isocrate ; elle figure explicitement dans la Rhétorique à Alexandre ${ }^{3}$.

Dans son état initial, l'exposé se présente sous la forme de la tripartition déjà mentionnée, le bref, le clair et le vraisemblable (ou « probable» / fiable) : $\beta \rho \alpha \chi \varepsilon ́ \omega \varsigma \kappa \alpha i ́$ $\sigma \alpha \varphi \tilde{\omega} \varsigma \kappa \alpha \grave{l} \mu \grave{\eta} \dot{\alpha} \pi \hat{\imath} \sigma \tau \omega \varsigma^{4}$, breuis-dilucida-ueri similis ${ }^{5}$ ou encore breuis-aperta-probabilis ${ }^{6}$, pour ne citer, comme équivalents latins, parmi beaucoup d'autres, que ceux de la Rhétorique à Herennius et du De l'invention: ces deux traités, dont les développements consacrés à la narration sont presque littéralement identiques, feront en cette matière figure d'autorité de référence pour la quasi-totalité de la théorie latine ultérieure, et leurs énoncés méritent donc à cet égard d'être mentionnés en toute première position.

Le schéma tripartite sera repris presque textuellement par Quintilien et les traités plus tardifs ${ }^{7}$. Il figure également, éventuellement augmenté de qualités supplémentaires diverses - mais non de la suauitas - dans les passages que les divers Progymnasmata consacrent à la narration, qu'il s'agisse, parmi les "exercices préliminaires», de la narration proprement dite, de la « fable » ou de la « chrie ${ }^{8}$ ».

6 La quadripartition, à savoir la tripartition précédente augmentée de la suauitas, a été transmise à l'humanisme essentiellement par l'intermédiaire des Divisions de l'art oratoire de Cicéron. La situation de cette quatrième «vertu " y est particulièrement complexe. En effet, en tant que qualité de la narration proprement dite, elle apparaît au chapitre IX (\$31-32), en compagnie de la clarté, de la brièveté et de la vraisemblance, et comme un élément ajouté (« sed adsumimus etiam suauitatem ", "accessoirement nous ajouterons l'agrément $\left.»^{9}\right)$. Elle s'y voit associer - non moins que les autres «vertus», d'ailleurs - toute une série de déterminations, selon un schéma général conforme à celui des traités antérieurs. Cependant, la même suauitas figurait déjà, mais en tant que qualité générale de l'expression, cette fois, à VI, 21-22, toujours précédée des trois "vertus" canoniques, dans une liste au demeurant augmentée d'une cinquième qualité, l'éclat ou illustris ( $\$ 20$, «Illustris est autem oratio», «On donne de l'éclat au style »), qui correspond à la fameuse notion stylistique grecque d' $\varepsilon \alpha^{\prime} \rho \gamma \varepsilon i \alpha^{10}$. En outre, la suauitas intervient encore, un peu plus loin, en XXI, 72-73, envisagée alors tant que qualité de l'éloge (voir Annexe, "Suauitas-Festiuitas: tableau comparatif»). Plus surprenant, quand Cicéron spécifie, avec plus ou moins de détails, les déterminations de la suauitas, considérée comme qualité générale du style, ou comme qualité de l'éloge, il met en avant, d'une part, des critères d'expression, se situant sur le plan des uerba, des mots, et, de l'autre, des critères de contenu, relevant donc des « choses » ou res : ce qu'il appelle, parlant de l'éloge, les « ornamenta rerum »- littéralement les « ornements des choses », ou, selon la traduction d'Henri Bornecque, «les ornements du fond », du contenu ${ }^{11}$. En revanche, quand Cicéron traite de la narration pure, il ne juge pas bon 
d'en mentionner d'autres. Comme si, en la matière, ces ornamenta rerum méritaient seuls d'être signalés. Non que les charmes de l'expression n'y revêtent pas d'importance, mais la force propre d'une narration suauis réside d'abord, ici, apparemment, pour Cicéron, en un certain art de la « mise en intrigue».

Si l'on se penche sur les « déterminations » de la suauitas, du moins celles qui visent le contenu (les ornamenta rerum), on constate un fait troublant. Outre qu'elles sont remarquablement concordantes entre elles, quel que soit celui des trois points de vue où elles sont envisagées dans les Divisions, on s'aperçoit qu'elles consonnent également de très près avec une autre liste de déterminations, figurant conjointement dans la Rhétorique à Herennius et le De l'invention: celle d'une autre "vertu », la festiuitas ${ }^{12}$, mot que Guy Achard, traducteur des deux ouvrages, rend par "agréable » ou " agrément » (il traduit de même suauitas ${ }^{13}$ ). Une nuance : ces deux traités, on l'a vu, ne retenaient que la liste canonique des trois virtutes; s'ils introduisent néanmoins en outre la festiuitas, c'est comme en annexe, en tant que qualité spécifique à un type précis de narration, celle qui porte sur les personnes (in personis), distinguée de celle qui porte sur les actions (in negotiis). Une question vient alors spontanément à l'esprit : suauitas et festiuitas ne seraient-ils pas deux équivalents distincts d'un seul et même terme grec ${ }^{14}$ ? L'hypothèse apparaît défendable: on l'a vu, les "vertus" du style, dans la théorie rhétorique antique, sont dotées d'un fort tropisme migratoire. Elles ont apparemment migré du statut de qualités de la narration vers celui de qualités du style en général; Cicéron faisait de la suauitas, successivement, une qualité générale du style, une qualité de la narration et une qualité de l'éloge. On ne voit donc pas ce qui aurait empêché, à un moment donné, la qualité originale grecque de se spécialiser, plus ou moins temporairement, dans la caractérisation plus étroite de la narration qui ne porte que sur les personnes ${ }^{15}$.

De quel terme grec le latin suauitas - comme éventuellement aussi festiuitas - est-il donc la traduction? La réponse est peu douteuse. Il doit s'agir du couple $\dot{\eta} \delta o v \dot{\eta}-\dot{\eta} \delta \dot{v} \varsigma^{16}$. En effet, Aristote dans sa Rhétorique polémique contre des théoriciens qui prétendent, au sujet de la $\lambda \varepsilon ́ \xi ı \varsigma$, du «style », "qu'il doit avoir de l'agrément et de la magnificence »,

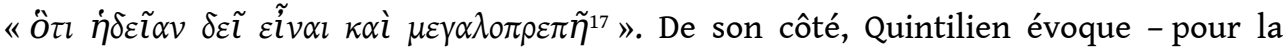
récuser - la position de Théodecte, qui «veut que le style de la narration ait non seulement de la magnificence, mais aussi de l'agrément ", "non enim magnificam modo uult esse, uerum etiam iucundam expositionem ${ }^{18}$ ". Le rapprochement, confirmé par d'autres sources, a induit la majorité des critiques à voir dans l' $\dot{\delta} \delta \varepsilon \tilde{l} \alpha \lambda \varepsilon^{\prime} \xi \varsigma$ (littéralement « le style agréable », l'agrément du style) unc contribution à la théorie de la narration propre à ce même Théodecte, contemporain, et semble-t-il, élève, d'Aristote, mais aussi d'Isocrate ${ }^{19}$.

9 Ce voisinage avec Aristote n'est pas sans intérêt, si nous nous reportons à la liste des ornamenta rerum, attachée à la suauitas aussi bien qu'à la festiuitas (voir Annexe: "Suauitas-Festiuitas : tableau comparatif »). On retrouve en effet dans cette liste en latin bien des éléments qui trouvent leur correspondant grec dans la Poétique ${ }^{20}$ :

- l'insistance générale sur les mouvements affectifs (dissimilitudo animorum, animorum motus,

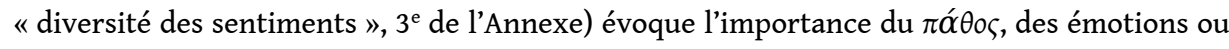
en grec des pathè ;

- le metus et la misericordia $\left(5^{\mathrm{e}}\right.$ et $\left.6^{\mathrm{e}}\right)$ font penser au couple fameux de la terreur et de la pitié :

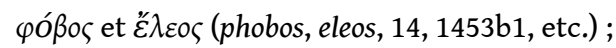

- la dissimulatio $\left(5^{\mathrm{e}}\right)$, au « $\psi \varepsilon v \delta \tilde{\eta} \lambda \hat{\varepsilon}^{\prime} \varepsilon \varepsilon v \dot{\omega} \varsigma \delta \varepsilon \tilde{l}$ » ou « façon dont on doit dire des mensonges ${ }^{21} »$; 
- la fortunae commutatio («changement de fortune », $7^{\mathrm{e}}$ ) n'est pas sans faire penser à la

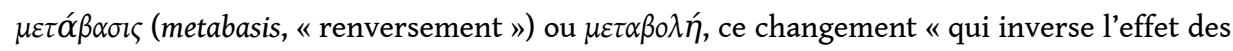

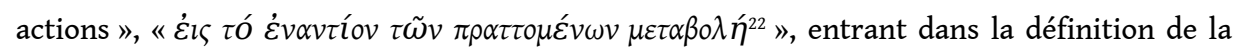
péripétie ou $\pi \varepsilon \rho \imath \pi \varepsilon ́ \tau \varepsilon l \alpha$;

- tout ce qui relève des effets de surprise (inopinata, insperata, improuisa, subita, " inattendue ", « imprévue ", " soudaine ", $8^{\mathrm{e}}$ et $9^{\mathrm{e}}$ ) répond à l'événement " contre toute attente ", « $\pi \alpha \rho \grave{\alpha}$ $\tau \grave{\eta} v \delta \circ \xi \not ́ \alpha v »(9,1452 \mathrm{a} 4$, para et doxa, d'où fr. paradoxe).

- enfin, l'admirabile ou les admirationes des Divisions de l'art oratoire ( $\$ 32$ et 73, «l'étonnement ", $1^{\mathrm{e}}$ de l'Annexe) pourraient correspondre au $\theta \alpha u \mu \alpha \sigma \tau o ́ v ~(24,1460 \mathrm{a} 11$, etc., thaumastos, « étonnant, merveilleux, extraordinaire »), etc.

Que cette constellation de procédés soit issue de la Poétique elle-même, ou qu'elle reflète une vulgate rhétorique antérieure, envers laquelle la Poétique serait redevable, il reste que la doctrine de la suauitas-festiuitas léguait à la rhétorique humaniste une conception de la narration étroitement apparentée à la mimésis aristotélicienne. Une rhétorique du récit qui est donc d'abord une poétique du muthos au sens de la Poétique, c'est-à-dire la «fable » au sens d' « histoire qui est racontée ", qu'elle soit légendaire ou $n^{23}$ : les ornamenta rerum sont une poétique du contenu. Mais aussi, à bien des égards, une rhétorique $d u$ "suspens ». C'est peut-être l'un des aspects par lesquels cette poétique implicite se distingue le plus de la stricte poétique aristotélicienne ${ }^{24}$ : elle met systématiquement en exergue toute une série de procédés narratifs de nature à « tenir en haleine » jusqu'au bout l'auditeur ${ }^{25}$. Aspect qui n'a pas manqué de retenir l'attention des humanistes, principalement à partir de la seconde moitié du XVI siècle.

\section{Les « vertus » de la narration dans la rhétorique humaniste}

11 La rhétorique occidentale a très longtemps admis comme seule norme doctrinale des «vertus » de la narration la tripartition «canonique » transmise par la Rhétorique à Herennius et le De l'invention, traités qui, de par leur caractère fondamentalement technique, se prêtaient mieux que d'autres à un type d'exploitation principalement scolaire. C'est évidemment le cas pour la rhétorique médiévale: le Livres dou tresor demande ainsi trois "choses", "que ele soit brieve, que ele soit clere, que ele soit voirsemblable ${ }^{26} »$; au début du $\mathrm{XVI}^{\mathrm{e}}$ siècle, pour Pierre Fabri, qui reprend largement Latini, la narration se doit encore d'être "clerement et intelligiblement proferee ", «brefve » et « vraye ou vray semblable ${ }^{27}$ ».

12 En dépit de la redécouverte progressive d'autres traités antiques, redécouverte proprement dite comme pour L'orateur et le De l'orateur de Cicéron, ou regain d'intérêt, en partie lié à une plus large diffusion, comme c'est le cas pour l'Institution oratoire ${ }^{28}$, l'attachement à la tripartition reste constant, jusques et y compris dans ce qui sera pour un temps la rhétorique officielle de la Compagnie de Jésus, le De arte rhetorica libri tres (1560) de Cipriano Soarez ${ }^{29}$.

13 Pourtant, aux alentours de 1560, nous voyons apparaître, d'abord timidement, la quadripartition, avec intégration de la suauitas. Le premier exemple que nous en ayons relevé se trouve dans les Institutionum rhetoricarum libellus de l'obscur Pierre SaintFleur, texte dédicacé à Jacques Amyot, cependant ${ }^{30}$. Saint-Fleur, à propos des " vertus " de la narration renvoie explicitement aux Divisions - ainsi qu'à Quintilien -, mais en se 
référant curieusement à leur $§ 21$, qui envisage la suauitas comme qualité générale du discours, et non pas spécialement de la narration, et en ne prenant en considération que sa partie stylistique, à l'exclusion des ornamenta rerum. Il n'en va plus de même dans les Ecclesiasticae rhetoricae (1576) de Louis de Grenade, traité qui bénéficiera d'une diffusion d'une importance exceptionnelle, et d'une influence proportionnée. Grenade, toutefois, n'utilise pas l'adjectif suauis, mais iucunda, c'est-à-dire le terme que nous avons vu Quintilien référer à Théodecte ${ }^{31}$ : "Jucunda vero narratio erit, si noua, inexpectata, magna, grauia contineat ${ }^{32}$ ", "La narration sera iucunda si elle comporte des choses extraordinaires, inattendues, importantes, sérieuses ». Évident amalgame des divers passages des Divisions relatifs à la suauitas ${ }^{33}$, qui, cette fois, met bien l'accent sur les res. Même remarque, mais avec mention explicite de la suauitas, dans une autre rhétorique ecclésiastique, celle du jésuite Lodovico Carbone (1590): "Quarta, quam aliqui tribus superioribus addunt, ut sit suauis et iucunda : cuiusmodi erit, si noua, si magna, si uaria, si grauia, continebit, et denique, si admirationes et expectationes habebit ${ }^{34}$ ", " Aux trois précédentes vertus, certains en ajoutent une quatrième, qu'elle soit suauis et iucunda : la narration le sera si elle comporte des choses extraordinaires, importantes, diverses, sérieuses, et enfin, des passages qui étonnent, d'autres qui captivent ". Carbone reprend presque sûrement Grenade, mais a toutes les chances de connaître directement aussi ses sources. Au même moment, Melchior Junius, professeur à Strasbourg, tient à peu près le même langage, tout simplement parce que sa liste recopie à l'identique celle du $\S 32$ des Divisions: "Suauis narratio est, quae habet admirationes, expectationes, exitus inopinatos, interpositos animorum motus ${ }^{35}$ ", "La narration est suauis quand elle a des passages qui étonnent, d'autres qui captivent, des dénouements inattendus, par endroits des mouvements pathétiques». Enfin, c'est encore vraisemblablement Grenade que plagie Vossius, professeur à Leyde, mais hostile au ramisme, en 1621: "Tertia uirtus ut sit suauis. Quod consequimur, si, quae narramus, sint magna, noua, inexspectata. Suauitatem quoque gignunt mores, dolores, metus; modo sine dicentis perturbatione proferantur. Alioqui enim amplificatio fuerit potius, quam narratio 36 , « La troisième vertu est d'être suauis. Ce qui se produira si nous narrons des choses importantes, extraordinaires, inattendues. La suauitas est produite aussi par l'ethos, la douleur, la crainte, mais pourvu que l'orateur les énonce sans s'émouvoir. Autrement en effet, ce serait plus une amplification qu'une narration. »

14 À partir du début du XVII ${ }^{e}$ siècle, donc, le schéma quadripartite avec suauitas est acquis, et il sera bien vite universellement adopté. Un bon témoignage de ce succès, non moins que de l'intérêt général suscité alors par la narration, est celui de Conrad Dieterich (1575-1639), dans ses Institutiones oratoriae de 1613 : il s'agit d'un théoricien d'obédience ramiste, courant qui, en principe, exclut la narration de la rhétorique proprement dite, pour la renvoyer à la dialectique, où elle est généralement assez vite expédiée. Dieterich, lui, traite spécifiquement de la narratio dans sa rhétorique, et propose la quadripartition (sous la forme breuis-perspicua-probabilis-suauis), augmentée toutefois d'un cinquième terme, "methodica ». On aura reconnu la vertu ramiste par excellence, la «méthode », dont le sérieux est sans doute appelé ici à contrebalancer la concession faite à la délectation, corollaire de la suauitas ${ }^{37}$.

15 Pour le reste, unanimes, Carlo Reggio, Caussin, Pajot, Pelletier, Hautin ${ }^{38}$, consacrent le triomphe de la suauitas, qui peut se présenter sous son aspect stylistique, également, mais intègre toujours désormais en complément la liste des ornamenta rerum. Les rhétoriques françaises ne sont pas non plus en reste. Pour René Bary, les narrations doivent être succinctes-claires-probables-excitantes ${ }^{39}$; excitante est manifestement un 
équivalent français de suauis, assez juste, en fin de compte. Pour Jacques Du Roure, il faut quatre "qualités » : clarté-vray semblance-agrément-brevetéte; l'ordre est bouleversé, mais la quadripartition est là, avec la suauitas, traduite cette fois par "agrément ". Bizarrement, Le Gras ne donne d'abord que la tripartition Clarté, Breveté, VraySemblance, mais il se hâte d'ajouter que la narration « doit avoir un air agreable, des surprises qui donnent de l'admiration ", "estre remplie de douceur et de gravité ", avec une liste de déterminations qui ne laisse pas de doute sur la présence en arrièreplan chez lui de la suauitas des Divisions, au demeurant contaminée avec la festiuitas ${ }^{41}$. Enfin, le succès de la suauitas ne manque pas de gagner les Progymnasmata: Pomey (1659) y introduit la quadripartition, et Jouvancy (1710) après lui, ne serait-ce que parce que, sur ce point, comme sur bien d'autres, il se contente de le recopier ${ }^{42}$.

Il convient de s'interroger sur les raisons qui, d'abord sur le plan matériel, ont présidé à la réapparition de la suauitas dans le canon des «vertus" de la narration en vigueur chez les humanistes. La plus évidente est la «redécouverte» des Divisions de l'art oratoire. Redécouverte qui n'est pas strictement matérielle : le texte n'avait jamais tout à fait disparu ; il suscite simplement un renouveau d'intérêt, qui n'est sans doute pas sans rapport avec la redécouverte, au sens strict, cette fois, des grands traités du Cicéron de la maturité, De l'orateur, Brutus, L'orateur. On sollicitera donc ici le témoignage de quelques grands commentaires renaissants des Divisions, commentaires dont on verra que certains ont pesé d'un poids décisif dans l'élaboration de la version humaniste de la suauitas.

Le premier est celui de Georges Valla ${ }^{43}$. Curieusement, le premier traducteur latin de la Poétique d'Aristote ${ }^{44}$ ne semble guère manifester d'intérêt pour la suauitas. Il annexe simplement aux $\S 21$ et 31 des Divisions de courts extraits de Cicéron supposés leur servir d'illustration.

18 Un intérêt plus affirmé s'esquisse dans les commentaires de Jacques-Louis d'Estrebay (1533 ou 1536) et de Jean Sturm (1545).

D'Estrebay accorde une certaine place à la suauitas, dont il analyse les procédés, mis en œuvre dans une narration des Verrines, en insistant sur l'extraordinaire (la nouitas) et le suspens: "Vbi principium audieris, illectus rei nouitate in longum uocabere, non sine expectatione iucunda, quid Rubrius sit facturus [...] tenebunt animum exitus inopinati $[. . .]^{45}$ ", «Dès que tu entendras le début de l'histoire, séduit par son aspect extraordinaire tu seras amené un long moment, non sans une délicieuse attente, à te demander ce que fera Rubrius [...] ton esprit sera tenu en haleine par les dénouements imprévus [...]». Des considérations du même ordre interviennent dans le commentaire sur le $\S 73$ des Divisions, D'Estrebay soulignant que « les événements tournent souvent autrement que ce à quoi s'attendait l'auditeur, dénouement imprévu qui procure un plaisir [uoluptas] plus agréable ${ }^{46} »$. Et d'illustrer le procédé par un exemple de l'Énéide, dont il cite les vers 740-741 du chant IX : Turnus encerclé par des ennemis en grand nombre, mais qui parvient en fin de compte à prendre le dessus.

Sturm va plus loin. Quand il paraphrase les § 31-32 des Divisions, comme D’Estrebay il redouble suaues par iucundae, mais il établit aussi, et surtout, un rapprochement avec la Poétique d'Aristote pour les données relatives aux laetitias, c'est-à-dire les passages qui suscitent la joie, l'allégresse : "quae non solum laetarum, sed etiam tristum rerum. Itaque illae $\pi \varepsilon \rho \imath \pi \varepsilon ́ \tau \varepsilon l \alpha l$ tragicae etiam suauitatem habent ${ }^{47}$ ", la laetitia ou joie "n'est pas seulement suscitée par les 'choses' joyeuses, mais aussi par les tristes : c'est pourquoi même les péripéties tragiques ont de l'agrément ». Le renvoi à la Poétique d'Aristote 
n'est pas moins évident pour le commentaire sur les $\$ 72-73$ des Divisions. Sur ce passage où Cicéron mentionne les " ornamenta rerum », Sturm intitule son commentaire "Generis demonstratiui uerborum \& rerum ornamenta ${ }^{48}$ ». Il introduit donc une distinction entre les "ornements de mots» et les "ornements de choses", expression qu'il reformule en « ornements de pensées » (sententiarum ornamenta) ${ }^{49}$. Abordant d'abord les "ornements des mots", il les subdivise encore, en recourant à la distinction cicéronienne ${ }^{50}$ entre mots "isolés » (uerba singula) et mots "agencés " (coniuncta ou collocata), qui lui sert à distinguer ce que nous appelons les "tropes" (translata i.e. métaphore, archaïsmes, etc.) des procédés relevant de la disposition des termes les uns par rapport aux autres (parallélisme, antithèse, etc., et composition périodique) ${ }^{51}$. Il revient alors au texte de Cicéron, et passe aux ornamenta rerum - opposés aux procédés principalement "verbaux » précédents -, ce qu'il reformule en une question (il s'agit d'un dialogue maître élève) : "Quae sunt illa sententiarum ornamenta? " "Quels sont ces " ornements de pensées » ${ }^{52}$ (dont parle Cicéron)? « Haec rebus ipsis ponderantur, in quibus est $\theta \alpha u ́ \mu \alpha \sigma l \varsigma$ admiratio, et $\pi \varepsilon p l \pi \varepsilon ́ \tau \varepsilon l \alpha$, euentus inopinatus ${ }^{53}$ ", "Ces derniers procédés, on les fait reposer sur les 'choses' elles-mêmes ${ }^{54}$, au nombre desquelles sont l'étonnement, en grec la thaumasis, et l'évènement imprévu, en grec la péripétie ".

21 Mais c'est surtout à partir du commentaire, posthume, de Majoragius (1514-1555), qu'éclate la mise en exergue de la suauitas ${ }^{55}$. Dans son commentaire du $\S 32$ des Divisions, Majoragius détaille en effet le contenu des uirtutes, en proposant pour chacune des quatre une liste numérotée de dix rubriques. Pour la suauitas (op.cit., fo $86 \mathrm{v}^{\circ}-87 \mathrm{r}^{\circ}$ ), ces dix rubriques sont, tout simplement, les dix déterminations du $\S 32$ des Divisions (cité dans l'Annexe), d'admiratio et expectationes à cupiditates. La présentation est ainsi très pédagogique : numérotées de 1 à 10 , les rubriques de la liste sont chacune illustrée par plusieurs exemples tirés des discours cicéroniens. Mais Majoragius ne se contente pas de reprendre la liste de déterminations fournies par le texte cicéronien. Il explicite chacune d'entre elles en exploitant largement les données fournies par les autres passages des Divisions. Ainsi, pour la rubrique 1, admiratio, quand il écrit "Primo admirationem, quae tum excitatur, cum aut magna, aut noua \& inaudita dicimus ${ }^{56}$ », on reconnaît l'emprunt au $\S 22$ des Divisions ${ }^{57}$ : «cum aliquid aut inuisum aut inauditum aut nouum dicas ", " en exposant des choses qui n'ont été ni vues ni entendues ou qui sont entièrement nouvelles" (trad. Bornecque). De même, pour la rubrique 2, expectationes, il souligne leur effet de suspens en s'inspirant $\mathrm{du}$ "in audiendo uoluptatem» au $\$ 73$ des Divisions, le «plaisir de l'auditeur»: «Secundo, expectationes concitatae multum delectant, cum dubitant auditores quo res sit euasura ", "En deuxième lieu, les attentes suscitées sont un grand délice [delectant], quand les auditeurs se demandent comment l'affaire va se terminer ».

Tout indique que c'est principalement du commentaire de Majoragius que se sont inspirés les artisans du "retour de la suauitas » au cœur des traités humanistes de rhétorique. Les parallèles textuels montrent que Grenade, Carbone et Vossius, au moins, lui sont redevables ${ }^{58}$. La mention par Carlo Reggio du suspens doit en dériver également: "si narrando suspendamus animos auditorum ad auiditatem reliqua percipiendi $i^{59}$ ", «si en narrant nous tenons en suspens l'esprit des auditeurs, avides de savoir la suite ». Quant à Caussin, il recopie purement et simplement Majoragius, comme viennent de le montrer Suzanne Duval et Lise Charles dans cette même livraison. Le reste aura coulé de source. 
23 Mais cette influence toute matérielle n'aurait sans doute pas suffi à amorcer une pareille réaction en chaîne, si le terrain n'avait pas déjà été labouré. Nous avons relevé des frémissements chez D'Estrebay et Sturm. On pourrait également mentionner la Poetica (1516) de François Dubois, qui fondait sur un commentaire amplifié des déterminations de la festiuitas dans le De l'invention une poétique narrative largement sous-tendue par la notion de suspens, encore informulée, toutefois ${ }^{60}$.

Plus important nous paraît être un autre précédent, celui du De inuentione dialectica (écrit en 1479) de Rodolphe Agricola ${ }^{61}$, traité dont l'influence majeure sur la rhétorique humaniste n'est plus à établir. Agricola ne parle pas directement de suauitas. Par ailleurs, il envisage la narration dans une perspective atypique, en la résorbant dans la catégorie plus générale d'expositio qu'il oppose à l'argumentatio, tout en précisant que les deux éléments se combinent et se complètent perpétuellement. Reste qu'il accorde également une place significative à la delectatio, pour laquelle il propose une série de ressorts, correspondant exactement aux déterminations de la suauitas telles qu'elles figurent dans les Divisions: "Mentem [...] delectant omnia quae magna, admiranda, inopinata, inexpectata, inaudita sunt $[. . .]^{62} »$, " Ce qui procure du plaisir à l'esprit [delectant], c'est tout ce qui est important, qui étonne, qui est inattendu, qui captive, qui est inouï»; "habeat oratio motus animorum, colloquia personarum, consilia, euentusque rerum inopinatos ${ }^{63}$ ", « il faut que le discours ait des passages pathétiques, des dialogues, des desseins inattendus, tout comme les événements qui en résultent ». Agricola avait également souligné la cohérence de l'intrigue et les effets d'attente, mais à propos du probabilis, cette fois, sur l'exemple du Pour Milon ${ }^{64}:$ le « fiable », nous l'avons souligné en commençant, est un autre nom pour dire la troisième qualité de la narration, plus satisfaisant à notre sens que celui de "vraisemblable». En somme, la Dialectique d'Agricola, moins strictement «logiciste» qu'il pourrait le paraître, préparait souterrainement la voie à l'accueil enthousiaste qu'allait réserver un peu plus tard la rhétorique humaniste de la narration, tant au suspens qu'à la suauitas.

\section{Le « retour de la suauitas »: une « vertu » nouvelle pour une nouvelle rhétorique?}

Quels que puissent avoir été les cheminements précis du « retour de la suauitas », et ses conditions matérielles, il est permis de se demander si l'adoption rapide, et généralisée, $\mathrm{du}$ «nouveau » canon des « vertus » de la narration ne traduit pas une mutation plus profonde de la perspective rhétorique, au moins en ce domaine, voire un « changement de paradigme ».

Pour essayer d'y voir plus clair, il ne nous paraît pas inutile de revenir sur le processus qui, dans l'Antiquité même, avait abouti à l'addition de l'hèdonè-suauitas à la liste précédemment acceptée des trois « vertus » de la narration.

On s'attachera ainsi d'abord aux analyses de Pierre Chiron ${ }^{65}$, qui souligne comment on est passé d'une liste restreinte liée aux conditions d'exercice de la parole démocratique, où l'on insistait sur la nécessité de ne pas parler "en dehors de la cause ", à une liste élargie, intégrant des exigences plus «littéraires »: il s'agirait d'une conséquence de l'éclipse de la parole civique au profit d'une prise de parole scolaire, liée aux pratiques de la déclamation. Un auteur comme le Pseudo-Démétrios, dans son Traité du style, représenterait à cet égard un tournant majeur, aux alentours de l'an $100 \mathrm{av}$. J.-C. Il est 
significatif que, même si cet auteur ne recourt pas directement à la qualité d'hèdonè, il associe l'importance, nouvelle, de l'enargeia à des pratiques de l'ordre du suspens ${ }^{66}$.

De son côté, se concentrant au départ sur la " vertu » de breuitas, Marie Formarier ${ }^{67}$ met en valeur l'évolution de la pensée rhétorique de Cicéron, depuis ses positions de jeunesse exposées dans le De l'invention, en direction d'une perspective, là encore, plus "littéraire ", qui intègre les données de la mimésis aristotélicienne, notamment à travers le recours à la suauitas ${ }^{68}$. Elle souligne le fait que cet élargissement, aux yeux de Cicéron, aboutit en réalité à donner plus de force persuasive encore au discours oratoire. La suauitas, ce serait donc la notion clef d'une "rhétorique généralisée », en particulier cicéronienne ${ }^{69}$.

Une transposition de ces analyses à la situation de la Renaissance ne nous paraît pas impossible, mutatis mutandis. On ne saurait parler de démocratie pour la période médiévale. Néanmoins, le cadre rhétorique y est marqué de tendances "technicistes " et "logicistes », qui mettent inévitablement l'accent sur l'argumentation proprement dite. En outre, l'usage quasi exclusif de la Rhétorique à Herennius et du De l'invention, sans parler des rhéteurs tardifs, ne pouvait qu'accentuer la tendance. Pour le Moyen Âge, la rhétorique, du moins la "rhétorique première ${ }^{70}$ ", reste fondamentalement un ensemble de techniques de persuasion. On n'aura donc pas de mal à diagnostiquer, derrière le recours à la suauitas, un "virage littéraire ", pris par l'humanisme, tout-àfait parallèle à celui qui avait été opéré dans l'Antiquité au début de l'âge hellénistique ${ }^{71}$.

Il n'est sans doute pas indifférent de noter que la «redécouverte de la suauitas » est exactement contemporaine de celle de la Poétique d'Aristote ${ }^{72}$. Georges Valla traduit la Poétique et commente les Divisions, même s'il n'en tire pas grand parti. Sturm opère des rapprochements entre les deux textes, parfaitement pertinents, à notre sens. La place centrale conférée par la suauitas aux ornamenta rerum est ainsi pleinement consonante avec le rôle essentiel du muthos (l'histoire qui est racontée) propre à la mimésis aristotélicienne. Les procédés de détail, nous l'avons vu, sont largement identiques. La suauitas, plus ou moins fusionnée avec la festiuitas, ce serait donc, pour la rhétorique humaniste, comme cela l'avait été pour Cicéron, si l'on suit M. Formarier, l'instrument majeur de l'intégration à la théorie de la narratio des ressources de la mimésis aristotélicienne. Il ne s'agit plus seulement désormais, au sens étroit, de convaincre, mais de captiver et de dégager du sens.

31 Il nous faut toutefois souligner encore cet aspect par lequel la poétique implicite de la suauitas se distingue malgré tout de celle d'Aristote: le suspens. Celui-ci n'intervient jamais explicitement dans la Poétique, il est même dans une certaine mesure récusé par la Rhétorique ${ }^{73}$. En revanche, il triomphe dans les interprétations que l'humanisme nous fournit de la suauitas; sous différentes formulations, nous avons vu le suspendere animos s'étaler, de Majoragius à Carlo Reggio, et au-delà : « tenir les esprits en suspens ", c'està-dire les tenir par l'incertitude de la suite des événements, les maintenir dans l'attente ou exspectatio.

On ne peut donc pas ne pas opérer un rapprochement avec les analyses proposées à ce sujet, dans un article qui a fait date, par Terence Cave ${ }^{74}$. Celui-ci pointait du doigt l'émergence de la notion de suspens, au milieu du XvI ${ }^{\mathrm{e}}$ siècle à travers, principalement, l'analyse de textes de la Poetica de Vida, de la préface aux Éthiopiques d'Amyot et des Poetices libri septem de J.C. Scaliger, qui, sur ce point, réfèrent au même roman ${ }^{75}$. Là encore, on relève un synchronisme étonnant avec les premiers succès de la suauitas. 
Sans parler des concordances d'expression, on soulignera que, pour Vossius, le modèle par excellence de la narratio suauis, ce sont justement les Éthiopiques: Vossius avait lu Scaliger, sans aucun doute, mais peut-être aussi Amyot ${ }^{76}$.

À bien des égards, donc, il nous semble que le « retour de la suauitas » signe l'acte de naissance d'une nouvelle rhétorique du récit, dans laquelle le rhétorique se voit résolument investi par le poétique, ce qui n'interdit pas que le poétique ne se trouve par contrecoup lui-même investi par ce renouvellement majeur du rhétorique. Fondamentalement travaillé par la mimésis et le suspens, franchement orienté dans le sens de la prise en compte des réactions de l'auditoire, le "nouveau récit ", suauis, festiuus, se veut au plus haut degré un récit « captivant ». Il convient pour cela d'abord qu'il s'y passe «des choses»: ornamenta rerum, des événements, peu banals, déconcertants, dans une trame narrative qui se prête à tout moment à des retournements improbables, mais toujours perçus par l'auditoire comme susceptibles de se produire, de préférence au dernier moment ${ }^{77} \ldots$ Le tout sur fond de vivacité d'évocation, saynètes prises sur le vif, dialogues animés, mouvements affectifs intenses... Nous n'allons pas réécrire les Divisions de l'art oratoire, ni leurs commentaires humanistes. Nous laisserons simplement le lecteur se demander à quoi tout cela lui fait penser.

Et nous pourrions enfin nous interroger sur la meilleure manière de traduire suauis en français. Depuis Jacques Du Roure (1662) et jusqu'à Henri Bornecque (1924), les rhétoriques françaises ou les traductions de Cicéron proposent souvent "agrément ", qui nous paraît trop faible, au regard de la force de la notion, telle qu'elle ressort de l'emploi qui en est généralement fait dans nos textes. Nous avons suggéré " captivant ", et, au sens du XVII e siècle, l'adjectif « excitant » utilisé par Bary ne nous paraitt pas mal trouvé. Mais nous nous tournerions bien vers un auteur plus précoce: il s'agit du Jacques Amyot de la préface des Éthiopiques. Renvoyant à Strabon, Amyot nous indique que « trois choses » sont requises dans les fictions poétiques, dont : « Tiercement [...] la fiction dont la fin est l'esbahissement, \& la delectation, qui procede de la nouvelleté des choses estranges, \& pleines de merveilles ». On croirait lire les Divisions et leurs commentaires ("Delectat etiam quicquid est admirabile... ») : nouvelleté pour noua, choses estranges pour inaudita, pleines de merveilles pour admirabile ou admiratio ${ }^{78}$. Même impression, un peu plus loin, dans la description de l'effet de suspens. Bref, le binôme «l'esbahissement, \& la delectation » fournirait un excellent équivalent $\mathrm{XvI}^{\mathrm{e}}$ siècle du latin suauitas, au sens où le terme est entendu par la rhétorique humaniste. On l'aura

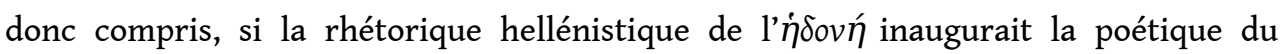

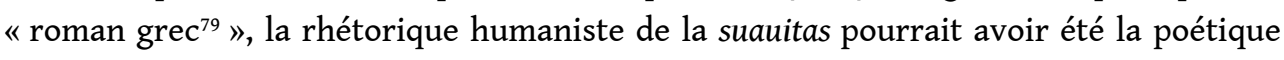
implicite du roman baroque ${ }^{80}$.

\section{ANNEXES}

ANNEXE 


\section{SUAUITAS - FESTIUITAS : TABLEAU COMPARATIF}

Il s'agit de comparer cinq passages : De l'invention (Inv. I, 27), la Rhétorique à Herennius (Her. I, 12), les Divisions de l'art oratoire (Part. 22, 32 et 72-73).

Nous mettons sous forme de tableau les trois passages qui sont très proches : Inv., Her. et Part. 32. Après le tableau, nous donnons le texte complet des cinq passages.

\begin{tabular}{|c|c|c|c|}
\hline & De l'invention, I, XIX, 27 & Rhétorique à Herennius, I, 12 & Divisions, IX, 32 \\
\hline & $\begin{array}{l}\text { Hoc in genere narrationis multa } \\
\text { debet inesse festiuitas, }\end{array}$ & $\begin{array}{l}\text { Illud genus narrationis quod in personis } \\
\text { positum est debet habere sermonis } \\
\text { festiuitatem }\end{array}$ & $\begin{array}{ll}\text { Suauis } & \text { autem } \\
\text { narratio } & \text { est, quae } \\
\text { habet } & \end{array}$ \\
\hline 1 & & & admirationes, \\
\hline 2 & & & exspectationes, \\
\hline 3 & $\begin{array}{l}\begin{array}{l}\text { confecta ex rerum uarietate, } \\
\text { dissimilitudine, }\end{array} \\
\text { animorum grauitate, lenitate, }\end{array}$ & $\begin{array}{l}\text { animorum dissimilitudinem, } \\
\text { grauitatem, lenitatem, }\end{array}$ & $\begin{array}{l}\underline{\text { interpositos }}-\underline{\text { motus }} \\
\underline{\text { animorum, }}\end{array}$ \\
\hline 4 & & & $\begin{array}{l}\text { colloquia } \\
\text { personarum }\end{array}$ \\
\hline 5 & $\begin{array}{l}\text { spe, metu, suspicione, desiderio, } \\
\text { dissimulatione, errore }\end{array}$ & $\begin{array}{l}\text { spem, metum, suspicionem, desiderium, } \\
\text { dissimulationem, }\end{array}$ & $\begin{array}{lr}\text { dolores, } & \text { iracundias, } \\
\text { metus, } & \text { laetitias, } \\
\text { cupiditates } & \end{array}$ \\
\hline 6 & misericordia, & misericordiam, & \\
\hline 7 & fortunae commutatione, & $\begin{array}{l}\text { rerum uarietates, fortunae } \\
\text { commutationem, }\end{array}$ & \\
\hline 8 & insperato incommodo, & insperatum incommodum, & \\
\hline 9 & $\begin{array}{l}\text { subita laetitia, iucundo exitu } \\
\text { rerum. }\end{array}$ & $\begin{array}{l}\text { subitam laetitiam, iucundum exitum } \\
\text { rerum. }\end{array}$ & exitus inopinatos \\
\hline & $\begin{array}{l}\text { Verum haec ex iis, quae postea } \\
\text { de elocutione praecipientur, } \\
\text { ornamenta sumentur. }\end{array}$ & $\begin{array}{l}\text { Verum haec in exercendo transigentur; } \\
\text { illud quod ad ueritatem pertinent, } \\
\text { quomodo tractari conueniat, aperiemus. }\end{array}$ & $\begin{array}{l}\text { Sed iam ad reliqua } \\
\text { pergamus. }\end{array}$ \\
\hline
\end{tabular}

Voici maintenant le texte continu correspondant au tableau, en traduction française pour Inv. et Her., en latin et français pour le $§ 32$ des Divisions (pour lequel, dans le tableau, nous avons changé l'ordre des rubriques). Nous donnons également le texte continu des deux autres passages des Divisions, les $\S 22$ et 72-73. Partout, nous ajoutons entre crochets droits les repères du tableau, de 1 à 9 ; les divers soulignements sont nôtres.

De l'invention, trad. G. Achard, op. cit., p. 83-84

I, XIX, 27 : «Ce genre de narration doit avoir beaucoup d'agrément, [3] grâce à la 
variété des événements ; à la diversité des sentiments : sérieux, douceur, [5] espoir, crainte, soupçon, désir, dissimulation, hésitation, [6] compassion ; [7] aux changements de fortune: [8] malheurs inattendus, [9] joies soudaines, heureux dénouement. Mais ces moyens seront tirés des préceptes que nous donnerons plus tard sur le style.»

Rhétorique à Herennius, trad. G. Achard, op. cit., p. 12-13

I, 12: «La narration qui est consacrée aux personnes doit présenter un style agréable, [3] des sentiments divers (sévérité, douceur, [5] espoir, crainte, soupçon, désir, dissimulation, [6] pitié) ; [7] des retournements de situation (changements de fortune, [8] malheur inattendu, [9] joie soudaine, heureux dénouement). Mais c'est en s'entraînant que l'on mènera à bien ces narrations. Nous allons montrer comment traiter ce qui se rapporte à des causes réelles. »

Divisions de l'art oratoire, trad. H. Bornecque, op. cit., p. 10-11

VI, 22: «Fit etiam suauis oratio, cum aliquid aut inuisum, aut inauditum, aut nouum dicas. delectat enim quicquid est admirabile [1]. Maximeque mouet ea, quae motum aliquem animi [3] miscet ${ }^{81}$, oratio. Quaeque significat oratoris ipsius amabiles mores, qui exprimuntur, aut significando ${ }^{82}$ iudicio ipsius $\mathrm{ex}^{83}$ animo humano, ac liberali, aut inflexione sermonis, cum aut augendi alterius, aut minuendi sui causa, alia dici ab oratore, alia existimari uidetur ${ }^{84}$ : idque comitate fieri magis quam uanitate."

«L'on donnera aussi de l'agrément au style en exposant des choses qui n'ont été ni vues ni entendues ou qui sont entièrement nouvelles. Ce qui plaît, en effet, c'est ce qui étonne [1], et le discours qui émeut surtout est celui qui provoque quelque émotion dans les âmes [3] et celui qui laisse voir que le caractère de l'orateur même mérite d'exciter la sympathie. Il produit cet effet, soit en marquant un jugement et un cœur humain et généreux, soit en usant d'un artifice de langage, quand pour élever quelqu'un ou se rabaisser lui-même, on sent qu'il dit d'une façon et pense d'une autre, et cela par courtoisie plutôt que par mensonge. "

Divisions de l'art oratoire, trad. H. Bornecque, op. cit., p. 15

IX, 32 : «Suauis autem narratio est, quae habet admirationes [1], exspectationes [2], exitus inopinatos [9], interpositos-motus animorum [3], colloquia personarum [4], dolores, iracundias, metus, laetitias, cupiditates [5]. Sed iam ad reliqua pergamus. "

«On trouvera de l'agrément aux narrations qui présentent des passages qui étonnent [1], qui captivent [2], des dénouements inattendus [9], par endroits des mouvements pathétiques [3], des dialogues [4], de la douleur, de la colère, de la crainte, de l'allégresse, des passions [5]. Mais voyons le reste. »

Divisions de l'art oratoire, trad. H. Bornecque, op. cit., p. 28-29

$\mathrm{XXI}, 72-73:$ : 72. et quoniam in his causis omnis ratio fere ad uoluptatem auditoris et ad delectationem refertur, utendum erit in is ornata oratione et singulorum uerborum insignibus quae habent plurimum suauitatis [...]. 73. Adhibendaque frequentius etiam illa ornamenta rerum sunt, siue quae mirabilia [1], nec ${ }^{85}$ opinata [9], siue significata monstris, prodigiis, oraculis, siue quae uidebuntur ei de quo agimus, accidisse ${ }^{86}$ diuina, atque fatalia. omnis enim expectatio [2] eius, qui audit, et admiratio [1] $]_{2}$ et improuisi exitus [9] habent aliquam in audiendo uoluptatem."

«72. Puisque, dans ces causes, on ne se préoccupe guère que de plaire à l'auditeur et de le charmer, on emploiera un style orné, des mots éclatants qui parent très agréablement le discours [...]. 73. Il faut aussi recourir assez fréquemment aux ornements de fond que voici : faits surprenants [1] et inattendus [9], ou bien annoncés par des phénomènes extraordinaires, des prodiges, des oracles, ou bien tels que les dieux et les destins semblent les avoir envoyés au personnage dont il s'agit. En effet l'attente [2] des auditeurs, l'étonnement [1], un dénouement imprévu [9] sont toujours pour eux une source de plaisir. » 


\section{NOTES}

1. L'idée même de redécouverte nous justifiera de ne pas traduire d'emblée le mot même de suauitas. L'un des enjeux de l'enquête est précisément de déterminer ce que les XVI ${ }^{e}$ et XVII ${ }^{e}$ siècles ont pu entendre par là. On nous permettra donc de ne proposer une traduction que dans notre conclusion, une fois le parcours achevé.

2. Lexis, « le style ». Voir P. Chiron, Un rhéteur méconnu : Démétrios (Ps.Démétrios de Phalère). Essai sur les mutations de la théorie du style à l'époque hellénistique, Paris, Vrin, 2001, p. 139.

3. Voir Pseudo-Aristote, Rhétorique à Alexandre, 30, 1438a-b, éd. P. Chiron, Paris, Belles Lettres, 2003, p. 79-82.

4. Ibid., p. 79 : brakheôs (sur brakhus), saphôs (sur saphès) et non-apistôs (autrement dit pistôs, adverbe formé sur pistis et pistos, « ce qui donne confiance », d'où " preuve », équivalents du latin fides dans « fidem facere », « convaincre »).

5. Rhétorique à Herennius (désormais Her.), I, 14, éd. G. Achard, Paris, Belles Lettres, 1989, p. 13.

6. Cicéron, De l'invention (désormais Inv.), I, Xx, 28, éd. G. Achard, Paris, Belles Lettres, 1994, p. 84.

7. Quintilien, Institution oratoire, IV, 2 ; pour les autres traités, voir nos « Matériaux pour l'étude de la théorie de la narration dans la rhétorique humaniste ".

8. Aelius Théon donne les trois qualités habituelles. Aphtonius ajoute la verborum proprietas, ou «propriété » des mots.

9. Cicéron, Divisions de l'art oratoire (désormais Divisions), IX, 31, dans Cicéron, Divisions de l'art oratoire, Topiques, trad. H. Bornecque, Paris, Belles Lettres, 1960, p.14; le §32 donne la tripartition sous la forme dilucide-breuitas-probabilis.

10. $\mathrm{NdE}$ - Sur l'enargeia, voir en dernier lieu les développements d'Amina Houara dans cette même livraison ("Démontrer l'évidence... », § 8-22).

11. Divisions, $\S 73$ (voir le texte dans l'Annexe). Le syntagme est aussi difficile à traduire que res lui-même. Guy Achard (Inv., I, XIX, 27) esquive la difficulté quand il rend ornamenta (du style ou elocutio) par «moyens» (du style), traduction qu'il justifie ainsi en note (op. cit., n. 82, p. 84 : « Les ornamenta ne sont pas nécessairement des embellissements, des ornements, mais des renforts de l'argumentation, des moyens de persuader ou d'intéresser (voir le sens premier d'ornare= équiper). » Lorsque Majoragius commente le $\S 73$ des Divisions ( f $^{\circ} 124 \mathrm{v}^{\circ}$ dans l'édition citée infra), il oppose les ornamenta rerum du texte cicéronien à l'exornatio uerborum: "Hactenus docuit, quomodo in exornatione verborum adhibeantur, nunc ad rerum ornamenta transit. ", " Jusqu'ici, Cicéron a traité de la façon dont on recourt aux procédés fondés sur les mots, maintenant il en vient aux procédés fondés sur les choses. »- Majoragius numérote ensuite les « ornements » qu'énumère le $\S 73$, des mirabilia aux fatalia, et il en fait ainsi sept rubriques.

12. Her., I, 13, p. 12-13; Inv., I, XIX, 27, p. 83.

13. Her., III, 22, p. 107 et IV, 69, p. 226.

14. Festiuitas devrait alors remonter à ce que l'on admet généralement comme étant la source latine commune au De l'invention et à la Rhétorique à Herennius (voir Her., éd. cit., p. XLVIII). Suauitas serait une re-traduction par le Cicéron de la maturité (celui qui écrit les Divisions de l'art oratoire). 15. Non sans rapport avec l'éloge, d'ailleurs. Nous laisserons de plus compétents que nous examiner le fond de la question. Pour la matière de cet article, il nous suffit de signaler que les rhétoriques humanistes, elles, ne cessent de contaminer suauitas et festiuitas, comme on le verra bientôt. Et les humanistes avaient souvent un sens très sûr de la pensée antique.

16. Hèdonè (d'où fr. hédonisme), "plaisir, jouissance, agrément ", et l'adjectif correspondant, hèdus. D'un point de vue étymologique, l'adjectif grec, hèdus, a comme "forme normalement modifiée » le latin suauis (Pierre Chantraine, Dictionnaire étymologique de la langue grecque, Paris, Klincksieck, 1999, p. 407).

17. Hèdeia, féminin de hèdus, et megalo-prepès, " qui a grand air » (sur prepô; les deux adjectifs qualifient la lexis) : Aristote, Rhétorique, III, 12, 1414a20, éd. M. Dufour et A. Wartelle, Paris, Belles 
Lettres, 1973, p. 76. Sur toute cette question, voir L. Calboli-Montefusco, «L'austera suavitas de l'orateur (Cicéron, De oratore, 3, 103)», Les noms du style dans l'Antiquité gréco-latine, textes présentés et édités par P. Chiron et C. Lévy, Peeters, Louvain, 2010, p. 113-129, p. 113.

18. Quintilien, Institution oratoire, IV, 2, 63, trad. J. Cousin, Paris, Belles Lettres, t. III, 1976, p. 56. On notera qu'on a affaire ici à un équivalent latin d'i் $\delta$ ć, iucundus, qui est différent de suauis autant que de festiuus.

19. Voir L. Calboli, op. cit., et P. Chiron, Un rhéteur..., op. cit., p. 139, note 264 et p. 145.

20. Le fait avait déjà été remarqué par Sturm. Voir infra.

21. Pseudos, «mensonge, fausseté » : Poétique, 24, 1460a19, trad. J. Lallot et R. Dupont-Roc (Paris, Seuil, 1980), p. 125.

22. Metabolè : ibid., 11, 1452a22, p. 71.

23. Ou «système des faits" ou «légende" (transmise par la tradition), selon J. Lallot et R. Dupont-Roc dans leur édition de la Poétique (op. cit., note p. 198-199 et entrée « histoire » de l'index des notions, p. 430).

24. L'autre est la faible insistance sur la nécessité de l'unité organique de l'histoire, capitale, en revanche, chez Aristote (Poétique, 7-8).

25. Sur l'émergence de cette tendance dans la rhétorique grecque, en lien intime avec l'insistance toujours plus forte sur l'enargeia, voir P. Chiron, Un rhéteur..., op. cit., p. 302-304, et « L'instruction "stylistique" dans la rhétorique gréco-latine ", Semitica et Classica, n 13, 2020, p. 79-104, p. 97. Aristote manifeste des réticences à l'égard de ces techniques, du moins dans le cadre de la narration oratoire (voir P. Chiron, Un rhéteur..., op. cit., p. 303, qui renvoie à Rhétorique $1415 \mathrm{a} 12$ $s q$.$) . Nous avons-nous même souligné cette dimension de la festiuitas dans notre dossier$ d'habilitation inédit sur la Poetica de François Dubois (1516), qui repose tout entière sur le passage afférent du De l'invention.

26. Brunetto Latini, Li livres dou tresor. Série 1 / par Brunetto Latini, publ. pour la première fois d'après les manuscrits de la Bibliothèque impériale, de la Bibliothèque de l'Arsenal, et plusieurs manuscrits des départements et de l'étranger par P. Chabaille, Paris, Imprimerie Impériale, 1863, III, I, XXXVIII, p. 519.

27. Pierre Fabri, Le grant et vray art de plaine rhetorique, éd. Héron, Rouen, Cagniard, 1889, p. 66.

28. De toute façon, on l'a vu, Quintilien adopte le schéma tripartite.

29. C. Soarez, De arte rhetorica libri tres. Ex Aristotele, Cicerone et Quinctiliano praecipue deprompti (1560), Brescia, T. Bozzola, 1581, fo $31 \mathrm{v}^{\circ}$ : «breuis, aperta, probabilis » ("brève, claire, fiable »). Soarez connait pourtant bien les Divisions, auxquelles il renvoie sans cesse, et dont il tire sa définition de la narratio.

30. Pierre Saint-Fleur, Institutionum rhetoricarum libellus, Paris, T. Brummenius, 1561, cap. XXI, $f^{\circ} 29 v^{\circ}$.

31. Voir supra.

32. Louis de Grenade, Ecclesiasticae rhetoricae (1576), Venise, A.Ziletti, 1578, p. 142 ; nous traduisons, ici et ensuite pour toutes les citations.

33. Auxquels il convient d'ajouter De l'invention, I, XVI, 23 (sur l'attentio dans l'exorde) : «Attentos autem faciemus si demonstrabimus ea quae dicturi erimus magna, nova, incredibilia esse ", "Nous rendrons les gens attentifs si nous leur montrons que ce que nous allons dire est important, extraordinaire, incroyable » (trad. G. Achard, op. cit., p. 78). L'amalgame avait déjà été opéré par Majoragius.

34. Lodovico Carbone, De causis eloquentiae libri IIII (1590), Venise, D. Zenario, 1593, p. 119.

35. Melchior Junius, Artis dicendi praecepta secundum oratorii officii partes breuiter ex Platone, Aristotele, Hermogene, Cicerone, Herenniano magistro, Quintiliano congesta ac digesta (1589), Strasbourg, A. Bertramus, 1607, p. 331 ; nous traduisons en reprenant la trad. Bornecque des Divisions. 
36. G. J. Vossius, Rhetorices contractae siue partitionum oratoriarum libri (1621), Leyde, J. Maire, 1640, p. $175-176$.

37. C. Dieterich, Institutiones oratoriae, sive De conscribendis orationibus..., Giessen, N. Hampel, 1613, cap. IV «Narratio», p. 18: «IV. Suauis, jucunda \& amabilis, ut cum delectationes \& admirationes quapiam audiatur», avec «1. uerba elegantia \& leniter cadentia [...], 2. affectibus et gestibus [...], 3. expectationes, admirationes, exitus inopinatos, colloquia ac jurgia personarum, \&c. ».

38. Pour le détail, voir nos «Matériaux pour l'étude de la théorie de la narration dans la rhétorique humaniste ".

39. R. Bary, La rhétorique françoise où pour principale augmentation l'on trouve les secrets de nostre langue (1653), Paris, Le Petit, 1660, p. 268.

40. J. Du Roure, Rhetorique françoise, Paris, Chez l'Auteur, 1662, p. 44.

41. Le Gras, La rethorique Françoise ou les preceptes de l'ancienne et vraye eloquence, Paris, Antoine de Raffle, 1671, p. 114. La contamination a été judicieusement décelée par J.-Y. Vialleton.

42. F. Pomey, Candidatus rhetoricae seu Aphthonii progymnasmata (1659), Munich, W. Schell, 1664, p. 258 ; J. de Jouvancy, L'élève de rhétorique (Candidatus rhetoricae, 1710, dernier manuel jésuite), éd. et trad. D. Denis et Fr. Goyet, Paris, Classiques Garnier, 2020 (reprend le texte latin de l'édition de Paris, Barbou, 1712 et la traduction française d'H. Ferté, 1892).

43. Preclarissimi viri Georgij Valle Commentationes. In Ptolomei quadripartitu[m] inq[ue] Ciceronis Partitiones [et] Tusculanas questio[n]es ac Plinij Naturalis historie librum secundu[m], Venise, Bevilacqua, 1502, bI r ${ }^{\circ}$ et bIII vo. Édition posthume, puisque Giorgio Valla, né en 1447, meurt en 1500.

44. Dont la traduction semble d'ailleurs ne pas avoir retenu beaucoup l'attention du public lettré, sur le moment.

45. Jacques-Louis d'Estrebay (Strebaeus), M. Tvl. Ciceronis, De partitione oratoria, dialogus, Iacobi Strebci..., Cologne, Gymnicus, 1539, p. 121. Sur D'Estrebay, voir l'étude classique de Kees Meerhoff (Rhétorique et poétique au XVI ${ }^{e}$ siècle en France..., Leyde, Brill, 1986), et en particulier son annexe I, «Bibliographie de Jacques-Louis d'Estrebay (Iacobus Lodoicus Strebaeus)», p. 349-358. Pour le De partitione oratoria, dialogus..., Meerhoff (p. 349) date la première édition de 1536 (Paris, Michel Vascosan, avec préface « datée de Paris, le 15 juin 1535 ») ; L. D. Green et J. J. Murphy (Renaissance rhetoric. Short-title catalogue 1460-1700, Burlington, Ashgate, 2006, p. 111, leur référence RR 864) la datent de 1533 (Paris, Robert Estienne, exemplaire à la British Library); ils signalent également (p. 120, RR 965) une édition de 1535 contenant le commentaire de D'Estrebay (De partitionibus oratoriis dialogus, Paris, Michel Vascosan, 1535 et rééd. 1538, exemplaires mentionnés dans le National Union Catalogue), que nous n'avons pu voir.

46. Ibid., p. 206 : «haec porro complent expectationem atque desiderium audientis: inducunt admirationem, eoque saepenumero perueniunt, quo ventum iri non suspicabatur auditor: qui exitus improuisus gratiorem iniicit uoluptatem $»$.

47. J. Sturm, In partitiones oratorias Ciceronis dialogi quatuor (1545), Strasbourg, Blasius Fabricius Chemnicensis, 1549, III, p. 168. Sturm n'insiste pas sur la composante de contenu du § 22. Voir V. Montagne, «Le De suaui dicendi forma de Jean Sturm: notes sur la douceur du style à la Renaissance ", Bibliothèque d'Humanisme et Renaissance, tome LXXVI, 2004, n 3, p. 541-563. V. Montagne s'intéresse aux tableaux fournis par Erythraeus, qui résument le commentaire de Sturm, en insistant sur le tableau correspondant aux § 21-22 des Divisions, qui ne porte pas sur la narration, donc. Erythraeus propose $\gamma \lambda v \kappa u ́ \tau \eta \varsigma$ (glukutès) comme équivalent grec de suauitas, sous l'influence d'Hermogène. Il passe toutefois à l'occasion à $\dot{\eta} \delta \varepsilon \tilde{l} \alpha$ (hèdeia, féminin de hèdus).

48. J. Sturm, In partitiones oratorias..., op. cit., IV, p. 271.

49. Il reprend cette formule, comme plusieurs éléments de son commentaire, d'un autre texte de Cicéron, L'orateur, XxxIX-XL, 134-139, dont le passage des Divisions est d'ailleurs plus ou moins un condensé. 
50. Ibid., 134 : «Nam et singulorum uerborum et collocatorum lumina attigimus", "En effet nous avons déjà fait allusion aux effets brillants qu'on tire des mots pris isolément ou en groupes. » (trad. A. Yon, Paris, Belles Lettres, 1964, p. 47).

51. Sturm suit L'orateur, XxxIx, 134-135. Her. nomme les figures stylistiques des «exornationes" (« exornationes sententiarum aut uerborum » à IV, 11).

52. On a vu plus haut que sententiarum ornamenta était synonyme pour Sturm d'ornamenta rerum. Sententia, terme fortement polysémique, prend alors le sens de "pensée», "contenu sémantique», plus large que celui de "phrase», "énoncé», qu'il a revêtu incidemment dans les lignes qui précèdent. Voir L'orateur, XXXIX, 136.

53. Ibid., p. 273. Sturm ajoute quelques explications, en mentionnant la mutatio fortunae (le changement de fortune, $7^{\mathrm{e}}$ de notre Annexe), ce qui montre qu'il a présents à l'esprit la festiuitas de la Rhétorique à Herennius et du De l'invention. Bref, Sturm a développé avant nous l'essentiel des hypothèses présentées plus haut.

54. Et non plus simplement sur les mots, isolés ou agencés ensemble.

55. Majoragius, M. Antonii Maioragii, commentarius in dialogum de Partitione Oratoria, Venise, Francesco de' Franceschi, 1587 (composé avant 1555 ; éditions posthumes, au moins à partir de 1569). Majoragius est très critique à l'égard de son prédécesseur D’Estrebay, désigné comme quidam Gallus, « un Français ».

56. $\mathrm{F}^{\circ} 86 \mathrm{v}^{\circ}$. À propos des $\S 21-22$, Majoragius précise simplement que « suauitas duplex est, altera verborum, altera rerum ", " la suauitas est double, l'une des mots, l'autre des choses » $\left(\mathrm{f}^{\circ} 67 \mathrm{v}^{\circ}\right)$ et indique que Cicéron traitera de la seconde plus loin, c'est-à-dire au $\S 31$. Pour le $\S 73$, sa présentation identique (sept rubriques avec des exemples, voir supra) est plus sommaire d'un point de vue théorique. Il y définit toutefois les «nec opinata " (les faits « inattendus ", son $2^{\mathrm{e}}, 9^{\mathrm{e}}$ de notre Annexe) comme "quae praeter expectationem et opinionem ab aliquo egregie fiunt ", " ce qui se fait de façon remarquable contre l'attente et l'opinion préétablie ».

57. Et au De l'invention, voir supra.

58. Voir supra: l'adjectif magna n'apparaît pas dans le texte original, mais seulement dans le commentaire de Majoragius, qui le tire, nous l'avons dit, des considérations de Cicéron sur l'attention dans l'exorde.

59. C. Reggio, op. cit., p. 537.

60. Voir supra.

61. R. Agricola, De inuentione dialectica libri omnes (1515), Cologne, Kempensis (Johann von Kempen), 1542. Majoragius connaît Agricola et le cite fréquemment, au même titre que Laurent Valla.

62. Ibid., III, 4 « De delectatione... », p. 435 ; éd. Lothar Mundt, Tübingen, Max Niemeyer, 1992, p. $458,1.41$.

63. Ibid., p. 439 (éd. Mundt, op. cit., p. 462, 1.106-107). Agricola rattache cette seconde liste, toutefois - à la différence de la première, qui relevait des res-, au genus orationis, au genre oratoire, puisque, selon lui, ces choses, qui en elles-mêmes ne sont pas agréables, le sont rendues par la façon de les raconter. Dans tous les cas, s'il n'a pas la formule d'ornamenta rerum, tout son chapitre s'oppose en bloc aux figures ou «ornements » de mots et de pensées («Ornamenta etiam omnia verborum, sententiarum » : fin du chapitre III, 4 : éd. Mundt, p. 462, 1. 146-147).

64. Ibid., p. 356 (éd. Mundt, op. cit., p. 350, 1. 77-85).

65. Communication personnelle de l'auteur. Voir aussi Un rhéteur..., op. cit., p. 133-136 et p. 371-372.

66. Voir supra.

67. M. Formarier, « La narratio chez Cicéron... », op. cit.

68. M. Formarier montre (op. cit., § 20) comment la festiuitas, dans le De l'orateur, en vient à gagner la totalité des narrations, et plus seulement la narration in personis, à laquelle la limitait le De l'invention. La festiuitas ne se distingue donc désormais plus guère de la suauitas, dont elle intègre 
même explicitement certaines déterminations : par exemple, le «coupé de dialogues » du De l'orateur (« interpuncta sermonibus », II, 328) correspond au « dialogues » des Divisions («colloquia personarum ", § 32, $4^{\mathrm{e}}$ de notre Annexe).

69. Formule reprise de P. Chiron, Un rhéteur..., op. cit., p. 136.

70. Par opposition à la « seconde rhétorique », qui est l'art du discours en vers.

71. Il ne faudrait pas minimiser la portée proprement « littéraire » des exposés consacrés à la narratio dans les premiers traités latins. De toute façon, la Rhétorique à Herennius (I, 12) aussi bien que le De l'invention (I, XIX, 27) distinguent trois genres de narration : a) portant sur l'objet de la controverse, b) digressif, c) « étranger aux procédures civiles ». Pour ce dernier genre, Her. ajoute qu'il est utile comme entraînement en vue des deux premiers, et Cicéron le caractérise comme "delectationis causa non inutili cum exercitatione dicitur et scribitur» (ce genre est « dit et écrit pour plaire, et permet de s'entraîner d'une façon qui n'est pas inutile », trad. G. Achard modifiée). On est donc là bien près de la narration « littéraire », surtout chez Cicéron. Par ailleurs, les deux traités fournissent des exemples littéraires : une comédie de Plaute, dans Her. (I, 15), des vers de Pacuvius, Ennius et Térence, chez Cicéron, pour illustrer chacune des espèces de la narration, fabula, historia et argumentum (Inv., I, XIX, 27: «le récit légendaire, l'histoire, la fiction », trad. Achard, op. cit., p. 83, qui cite en note les équivalents grecs donnés par Sextus Empiricus, muthos, historia, plasma) ; Her. de son côté rapproche la fabula de la tragédie et l'argumentum de la comédie (I, 13). On ne s'étonnera donc pas que ce tropisme " littéraire ", déjà plus affirmé au départ chez Cicéron que dans Her., dont il n'est toutefois pas absent, n'ait pas cessé de s'accentuer au fur et à mesure de la carrière de l'Arpinate (voir M. Formarier, art. cit.). Nous remercions le groupe RARE pour sa contribution à ces remarques.

72. Principalement diffusée, on le sait, à dater de la traduction et du commentaire de Robortello (1548).

73. Voir supra.

74. T. Cave, "Suspendere animos : pour une histoire de la notion de suspens ", in Les commentaires et la naissance de la critique littéraire en France, dir. G. Mathieu-Castellani et M. Plaisance, Paris, Aux amateurs de livres, 1990, p. 210-218, repris dans Pré-histoires : textes troublés au seuil de la modernité, Genève, Droz, 1999, p. 129-141.

75. On pourrait en réalité relever quelques autres précédents, notamment antiques. Voir nos "Matériaux pour l'étude de la théorie de la narration dans la rhétorique humaniste ", en particulier pour les commentaires de Térence. Tout cela ne remet pas en cause la pertinence globale de l'analyse, pensons-nous.

76. On note que l'une des premières réapparitions de la suauitas se fait sous la plume d'une relation d'Amyot, Pierre de Saint-Fleur (voir supra).

77. Cicéron, au $\S 73$ des Divisions, fournit une indication intéressante, souvent relevée par les commentateurs : "siue quae uidebuntur ei de quo agimus accidisse divina atque fatalia ", " ou bien tels que les dieux et les destins semblent les avoir envoyés au personnage dont il s'agit» (trad. H. Bornecque). Il convient que, derrière l'enchaînement des événements, l'auditoire pressente quelque chose comme une intentionalité mystérieuse. Ce pourrait être là le ressort souterrain de l'admiratio, du $\theta \alpha u \mu \alpha \sigma \tau o ́ v . ~ B r e f$, pour captiver le public, il faudrait que les «choses» ne se présentent plus simplement comme des faits, mais accèdent à la condition de « signes ».

78. Amyot cite Strabon, lequel cite en fait Polybe traitant d'Homère et sa « liberté poétique, qui dose différemment l'information [historia], la mise en œuvre [diathesis] et la fable [muthos]. L'information vise à la vérité [...] ; la mise en œuvre vise à l'effet [energeia] (quand il introduit des récits de bataille); la fable vise à produire plaisir [hèdonè] ou terreur [ekplêxis]» (Strabon, Géographie, I, 2, 17, trad. G. Aujac, Paris, Belles Lettres, t. I, 1969, p. 104-105). Chez Strabon /

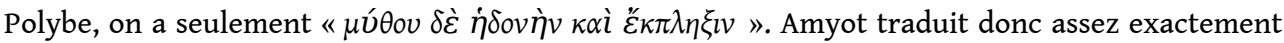
ce binôme plaisir et terreur, mais les spécifications qu'il ajoute sont de son cru, c'est-à-dire, très vraisemblablement du «nôtre », les Divisions et leur mouvance. Chez Polybe, on le voit, hèdonè 
appelait déjà un complément, l'épouvante ou «étonnement " produit par un choc : ekplêxis, sur plèssô, «frapper », se trouve dans la Poétique (16, 1455a17, op. cit., p. 91) pour dire «le choc de la surprise » dans le cas de la reconnaissance ou anagnôrisis (voir aussi Longin, Traité du sublime, 15.2, en poésie « le but est l'étonnement ", ekplêxis, et Démétrios, Du style, 283, éd. P. Chiron, Paris, Belles Lettres, 1993, p. 77, «tout ce qui frappe est véhément [deinon], parce qu'effrayant»). Ce complément va dans le sens des effets de sidération décrits plus tard par les traités comme caractéristiques de la suauitas.

79. Voir P. Chiron, cité supra.

80. Voir L. Plazenet, L'ébahissement et la délectation : réception comparée et poétique des romans grecs en France et en Angleterre aux XVI $I^{e}$ et XVII siècles, Paris, Honoré Champion, 1997 ; S. Duval, La prose poétique du roman baroque (1571-1670), Paris, Garnier, 2017. On remarquera cependant que dans son Projet de l'éloquence royale, Versailles / Paris, Ph.-D. Pierre / Lamy, 1805, p. 37-38, Amyot ne retient que les trois vertus canoniques, et ne mentionne pas la suauitas. Peut-être a-t-il estimé que cette qualité « romanesque »-non moins que l'usage de l'ordo artificialis - ne s'accordait pas avec la « gravité » convenable à l'éloquence du Prince.

81. Miscet est le texte que donne Majoragius (ici et ensuite, nous reprenons le texte et la ponctuation de son commentaire des Divisions). L'éd. Bornecque donne : ciet.

82. Bornecque : signando.

83. Bornecque : et.

84. Bornecque : uidentur.

85. Bornecque : rerum, siue admirabilia siue nec.

86. Bornecque : cecidisse.

\section{AUTEUR}

\section{JEAN LECOINTE}

Poitiers Forellis B 2 